\title{
Sample Asymmetry Analysis of Heart Rate Characteristics with Application to Neonatal Sepsis and Systemic Inflammatory Response Syndrome
}

\author{
BORIS P. KOVATCHEV, LEON S. FARHY, HANQING CAO, M. PAMELA GRIFFIN, \\ DOUGLAS E. LAKE, AND J. RANDALL MOORMAN
}

\begin{abstract}
Department of Psychiatric Medicine [B.P.K.], Department of Health Evaluation Sciences [B.P.K.], Department of Internal Medicine [L.S.F., H.C., J.R.M.], Department of Pediatrics [M.P.G.], Department of Mathematics [D.E.L.], Department of Molecular Physiology and Biological Physics [J.R.M.], and the Cardiovascular Research Center, University of Virginia Health System, Charlottesville, Virginia, 22908,

U.S.A.
\end{abstract}

\section{ABSTRACT}

We introduce the sample asymmetry analysis (SAA) and illustrate its utility for assessment of heart rate characteristics occurring early in the course of neonatal sepsis and systemic inflammatory response syndrome (SIRS). Conceptually, SAA describes changes in the shape of the histogram of RR intervals that are caused by reduced accelerations and/or transient decelerations of heart rate. Unlike other measures of heart rate variability, SAA allows separate quantification of the contribution of accelerations and decelerations. The application of SAA is exemplified by a study comparing 50 infants, who experienced a total of 75 episodes of sepsis and SIRS, with 50 control infants. The two groups were matched by birth weight and gestational age. RR intervals were recorded for all infants throughout their course in the Neonatal Intensive Care Unit. The sample asymmetry of the RR intervals increased in the $3-4 \mathrm{~d}$ preceding sepsis and SIRS, with the steepest increase in the last $24 \mathrm{~h}$, from a baseline value of $3.3(\mathrm{SD}=1.6)$ to $4.2(\mathrm{SD}=2.3), p=0.02$. After treatment and recovery, sample asymmetry returned to its baseline value of $3.3(\mathrm{SD}=1.3)$. The difference between sample asymmetry in health and before sepsis and SIRS was mainly due to fewer accelerations than to decelerations. Compared with healthy infants, infants who experienced sepsis had similar sample asymmetry in health, and elevated values before sepsis and SIRS $(p=0.002)$. We conclude that SAA is a useful new mathematical technique for detecting the abnormal heart rate characteristics that precede neonatal sepsis and SIRS. (Pediatr Res 54: 892-898, 2003)

$\quad$ Abbreviations
SAA, sample asymmetry analysis
HRC, heart rate characteristics
BW, birth weight
GA, gestational age
HR, heart rate
SIRS, systemic inflammatory response syndrome

Approximately 40,000 very low birth weight infants $(<1500$ g) are born in the United States each year (1). Survival for this

Received June 3, 2002; accepted May 22, 2003.

Correspondence: Boris P. Kovatchev, Ph.D., Box 800137, University of Virginia Health System, Charlottesville, VA 22908, U.S.A.; e-mail: boris@virginia.edu

Supported by National Institutes of Health grants R01-DK51562 and GM64640; the American Heart Association, Mid-Atlantic Consortium; Children's Medical Center Research Fund, University of Virginia; W.S. Paley Foundation; Virginia's Center for Innovative Technology; and Medical Decision Networks, Charlottesville, VA.

Potential for conflict of interest: Medical Decision Networks of Charlottesville, VA, which supplied partial funding for this study, has a license to market technology related to heart rate characteristics (HRC) monitoring of newborn infants. As of the submission date of the final version of the article, none of the authors had received consultants' fees or owned equity in Medical Decision Networks or related companies. However, Drs. Griffin and Moorman have been offered an equity share of a new company, Medical Predictive Systems Corporation, that owns the HRC technology license.

DOI: 10.1203/01.PDR.0000088074.97781.4F group has improved with advances in neonatal intensive care, but late-onset sepsis continues to be a major cause of morbidity and mortality $(2,3)$. The clinical syndrome of sepsis and SIRS is brought about by the host response to insults such as bacterial infection, and has been named the $\operatorname{SIRS}(4,5)$. Neonatal sepsis occurs in as many as $25 \%$ of infants weighing $<1500 \mathrm{~g}$ at birth (2), and the rate is about 1 per 100 patient days $(6,7)$. The National Institute of Child Health and Human Development Neonatal Research Network found that neonates who develop late-onset sepsis have a $17 \%$ mortality rate, more than twice the $7 \%$ mortality rate of noninfected infants, as well as increased morbidity (2).

Unfortunately, early diagnosis of neonatal sepsis is difficult, as the clinical signs are neither uniform nor specific (8). 
Clinical neonatologists recognize sepsis as a potentially catastrophic illness, and obtain blood cultures and administer antibiotics empirically for subtle symptoms in an attempt to avert disaster. This leads to unnecessary blood cultures and short courses of antibiotics to infants without bacterial infection - in fact, 10 to 20 infants are treated for sepsis for every one infant that has a positive blood culture (9). Although the blood culture is felt to be the gold standard for establishing the diagnosis of sepsis, there are concerns regarding its reliability, especially if single samples of small volume are submitted, as is often the practice in critically ill newborn infants $(10-12)$.

Because HRV, which arises from the interplay of the sympathetic and parasympathetic arms of the autonomic nervous system, is abnormal in many disease states, we hypothesized that a successful surveillance strategy for sepsis in neonates might be based on quantitative HR monitoring. Indeed, we found that infants destined for sepsis and SIRS developed patterns of HR that were similar to those of fetal distress, with reduced variability and transient decelerations (13). Because these changes are not reported by conventional means of HR analysis, we developed measures based on moments and percentiles of the data. We found significant dynamic differences in these HRC measures in selected high-risk infants who developed sepsis and SIRS compared with lower-risk control infants who did not. Particularly intriguing and promising was the observation of a marked asymmetry of the distribution of RR intervals before illness that was evident on inspection of frequency histograms. We investigated the ability of standard measures of asymmetry, such as the third moment, or skewness, of the distribution to relate to the HR abnormality. Like all higher moments, though, skewness reports on deviations from the mean value, which is not an accurate measure of the center of asymmetric distributions. Because we are most interested in asymmetric distributions, we sought to develop a new measure that reported on deviations around a more realistic center. In addition, we sought a means to distinguish the contributions of HR accelerations and decelerations to the characteristic abnormality.

Here, we introduce the SAA, a general technique to quantify the asymmetry of frequency histograms with respect to their mean, median, or any other empirically derived point. We validate its use for detecting the abnormal HRC before neonatal sepsis in a new clinical database, and find that an absence of HR accelerations is a more pronounced change than the presence of decelerations. This observation allows new insights into the pathophysiology of HR control during illness.

\section{METHODS}

Subjects. To illustrate the utility of SAA, we retrospectively studied prolonged (duration of at least a week) recordings of
HR in 158 infants admitted consecutively to the Neonatal Intensive Care Unit at the University of Virginia Hospital whose parents gave consent. Fifty of these infants (experimental group) had a total of 75 episodes of sepsis and SIRS, defined to be present when a physician suspected sepsis and SIRS, obtained a blood culture, and administered antibiotic therapy for 7 or more days. A control group of 50 healthy infants was randomly selected from the remaining 108 consecutive admissions to precisely match the experimental group by BW and GA (Table 1). Because the entire group of healthy infants had on average higher BW and GA, the random selection of control infants used a nonuniform random generator with a higher probability of selecting low BW infants and lower probability of selecting higher BW infants (more precisely, the probability of selecting a control infant ranged from 1 for $\mathrm{BW} \leq 656 \mathrm{~g}$ to 0.16 for $\mathrm{BW}>1451 \mathrm{~g}$; these values were determined by the relative distribution of $\mathrm{BW}$ in the experimental versus control group). Once experimental versus control infants were matched by BW, the matching by GA occurred naturally (Table 1). The Human Investigations Committee of the University of Virginia approved the protocol for data recording.

Procedure. From the EKG signal of the bedside monitors, we recorded RR intervals in series of 4096 RR intervals (approximately 20-30 min of data) as previously described (13) and then summarized 6-h periods beginning at midnight with the median values of all measures. The final step of data preprocessing was to aggregate the data of all experimental and control infants into 24-h blocks for analysis. For each infant in the experimental group we tracked in days the time to sepsis and the time postsepsis, as well as the duration of antibiotic treatment. The data from the treatment days were excluded from the analysis. Thus, for each experimental infant, we had three sets of 24-h blocks of data: before sepsis (defined as $>1$ $\mathrm{d}$ before an episode), immediately before sepsis (within $24 \mathrm{~h}$ before an episode), and post-treatment (after the completion of antibiotic therapy) timed backwards from an episode of sepsis, or forward from the completion of treatment. For the control group, these blocks represented the average $24-\mathrm{h}$ period based on a week of observation selected at random.

Sample asymmetry analysis. We repeatedly observed that clinical events were associated with a marked asymmetry of the frequency histograms of RR intervals with large deviations to the right of the distribution median (13), the consequence of long RR intervals during HR decelerations, and only small deviations to the left, the consequence of fewer and smaller HR accelerations. Thus, quantifying the asymmetry of the magnitude of RR intervals with respect to their mean, median, or another empirically derived point is an important exercise in characterizing HRV samples. Previously, we used the classical

Table 1. Study population

\begin{tabular}{lccc}
\hline & $\begin{array}{c}\text { Experimental group: } \\
\text { episode(s) of sepsis } \\
\text { and SIRS }\end{array}$ & $\begin{array}{c}\text { Control group: } \\
\text { no episodes }\end{array}$ & $\begin{array}{c}\text { Group comparison } \\
(t \text { test })\end{array}$ \\
\hline Infants & 50 & 50 & - \\
Mean birth weight (SD) (g) & $1227(847)$ & $1228(760)$ & $t=0.01, p>0.99$ \\
Mean gestational age (SD) (d) & $198(34)$ & $196(26)$ & $t=0.37, p=0.72$ \\
\hline
\end{tabular}


distribution skewness to quantify this observed phenomenon (13). The skewness of a distribution of a random variable is the quotient of the third moment about the mean of the random variable and the third power of its $\mathrm{SD}$. The skewness is 0 for symmetric distributions, positive if the distribution has a longer tail to the right, and negative if the distribution has a longer tail to the left. However, skewness can represent only asymmetry around the distribution mean, which does not accurately report the center of asymmetric distributions, and does not allow separate quantification of HR accelerations (deviations less than the central RR interval, forming the left-hand portion of the histogram) from decelerations (deviations greater, forming the right). In addition, skewness does not allow for adjustment of the sensitivity of analysis (weighting) to large deviations. Figure 1 illustrates a positively skewed distribution with a longer right tail. The mean is greater than the median and poorly identifies the center of the distribution.

To overcome these limitations, we have developed the sample asymmetry - a new measure quantifying asymmetry of random variable distributions. The strict mathematical definition of sample asymmetry and the computational formulas used by SAA of a random variable are given in the Appendix. The important features of SAA are 1) deviations from the median (or any other arbitrary central point) are calculated, 2) there is separate quantification of deviations less than and greater than the central point, and 3) the weighting of deviations less than and greater than the central point can be separately adjusted by varying weighting parameters, i.e. the sensitivity of the analysis can be directionally increased, or decreased. The parabola in Figure 1 represents the weights assigned to left- and rightsided deviations from the median. Here we use sample asymmetry computed around the median of the RR intervals with weighting parameters $\alpha=\beta=2$ (see Appendix). We also examine the left- and right-weighted deviations $\mathrm{R}_{1}$ and $\mathrm{R}_{2}$, representing HR accelerations and decelerations, respectively.

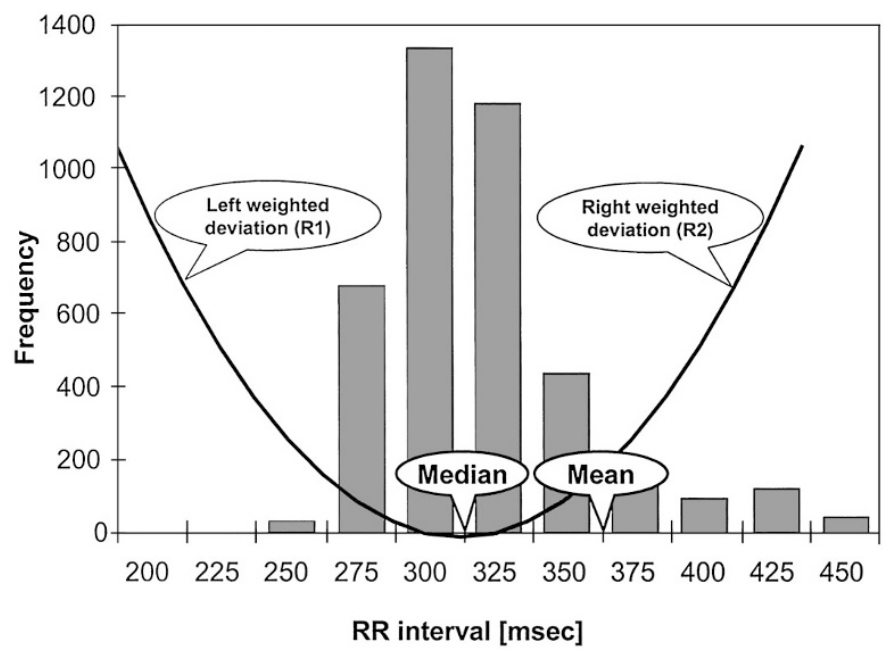

Figure 1. A positively skewed distribution with a shorter left tail and a longer right tail. The mean is greater than the median and poorly identifies the center of the distribution. The curve (a parabola) represents the weighting of left- and right-sided deviations of the median (with weighting parameters $\alpha=\beta=2$ ). The left branch $\mathrm{R}_{1}$ represents accelerations, e.g. lower $\mathrm{R} 1$ entails absence of accelerations; higher $\mathrm{R}_{2}$ means more decelerations.
Data simulation. We simulated the neonatal HR data by adding two signals (14). The first, which represents normal neonatal HRV, was a stationary time series with the same frequency content as normal neonatal HR records. It was calculated as the inverse Fourier transform of the spectrum of a random sample of clinical data obtained during health. The second, which represents transient decelerations, was a clinically observed deceleration of duration $50 \mathrm{RR}$ intervals that occurred just before an episode of sepsis. We added scaled versions of this deceleration, and plotted SAA parameters as a function of the height (in multiples of the SD of the baseline) and number of added decelerations. Each simulated data set had 4096 points, and we analyzed 100 realizations for each condition tested.

Statistical analysis. Group comparisons of BW and GA were performed using $t$ tests. To compare sample asymmetry in periods before sepsis, immediately before sepsis, and posttreatment in experimental-group infants, we used repeated measures ANOVA with two contrasts: 1) comparing periods of health ( $5 \mathrm{~d}$ before sepsis) versus immediately before sepsis, and 2) comparing the two periods of health, $5 \mathrm{~d}$ before sepsis versus post-treatment. ANOVA with covariates BW and GA was used to compare control versus experimental-group infants, the latter group restricted to periods without sepsis. Calculations used SPSS (SPSS Inc., Chicago, IL, U.S.A.).

\section{RESULTS}

Example of the analysis of clinical data. The plots in Figure 2 are taken from the same infant, and each show 4096 consecutive RR intervals, or approximately $20-30 \mathrm{~min}$ of data. The infant was born 5 mo previously at 24 wk gestation and weighed $720 \mathrm{~g}$ at birth. The infant had evidence of chronic lung disease but was clinically stable at the time of the data in Figure 2. The data in panel $A$ were recorded remote from clinical events. The data in Figure 2, $B$ and $C$, were recorded 18 and $9 \mathrm{~h}$ before an acute clinical deterioration, and 30 and $21 \mathrm{~h}$ before death, respectively. The clinical differential diagnosis included septic shock, and blood cultures were positive for coagulase-negative Staphylococcus and a urine culture grew Escherichia coli.

The record in Figure $2 A$ represents normal HRV. As expected, there are incessant small changes in RR intervals associated with accelerations (shorter RR intervals) and decelerations (longer RR intervals). The abnormal records in Figure 2, $B$ and $C$, display no small or frequent changes. Rather, there is a long-lived baseline of very reduced variability punctuated intermittently by large decelerations. Hence, we describe the characteristic HR abnormality that occurs early in the course of neonatal sepsis and SIRS (13) as reduced baseline variability and transient decelerations. How do we quantify these changes? None of the decelerations would have triggered an alarm for low HR, which is conventionally set for 100 beats per minute (RR interval $600 \mathrm{~ms}$ ). Moreover, neither the mean RR intervals of the three data sets $(369,398$, and $362 \mathrm{~ms})$ nor the standard deviations $(3.7,5.0$, and $7.4 \mathrm{~ms})$ would identify the abnormal records. 


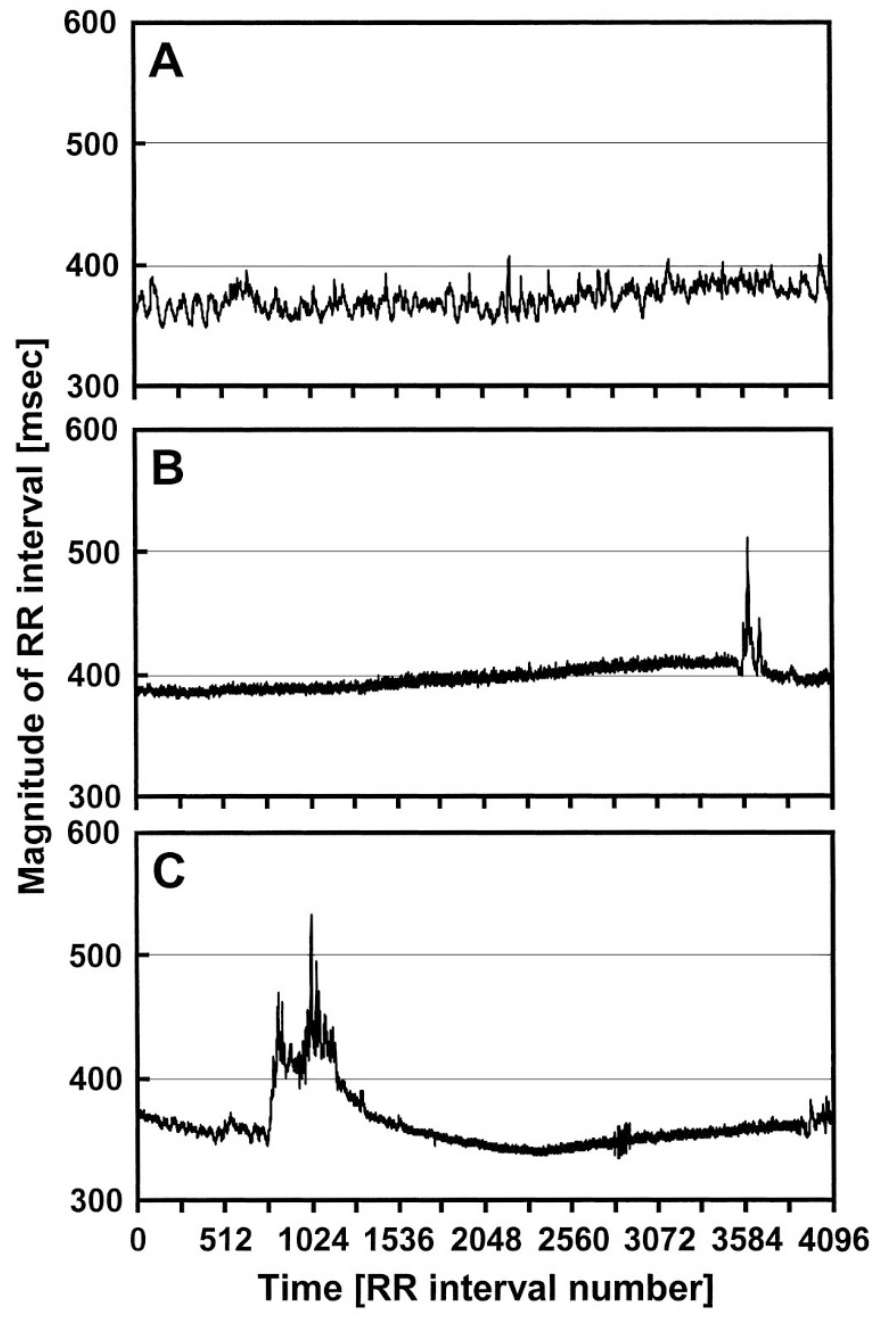

Figure 2. RR interval time series from the same infant while clinically stable $(A)$ and from $18(B)$ and $9(C) \mathrm{h}$ before an acute clinical deterioration leading to death. The abnormality before illness is reduced variability and transient decelerations that do not reach RR intervals of $600 \mathrm{~ms}$ necessary to trigger bradycardia alarms. Note that the normal record in $A$ is marked by frequent small accelerations and decelerations, but that these are both absent in the records in $B$ and $C$. Only infrequent and large decelerations occur before illness. These observations prompted our investigation of the symmetry of the distributions of RR intervals.

The corresponding frequency histograms of these time series data are shown in Figure 3. The observed abnormality causes marked asymmetry of the histograms in Figure 3, $B$ and $C$, with a reduction of the magnitude of shorter-than-median RR intervals and a clearly right-skewed distribution. These abnormalities can be assessed by increased skewness ( 0.82 for Fig. $3 A$ compared with 1.92 and 1.55 for Fig. 3, $B$ and $C$ ).

We quantified the observed asymmetry using our sample asymmetry algorithm with weighting parameters $\alpha=\beta=2$ (see Appendix). In Figure 3, the parabolic weighting functions corresponding to these parameters are superimposed over the RR interval histograms. The values of the sample asymmetry $R$ $(2,2)$ are 1.37 for the data in Figure $3 A$, and 2.97 and 11.8 for the data in Fig. 3, $B$ and $C$. The values of the weighed deviations $R_{1}$ and $R_{2}$ are presented as well. In this example, sample asymmetry increased dramatically before the clinical diagnosis of sepsis and SIRS.

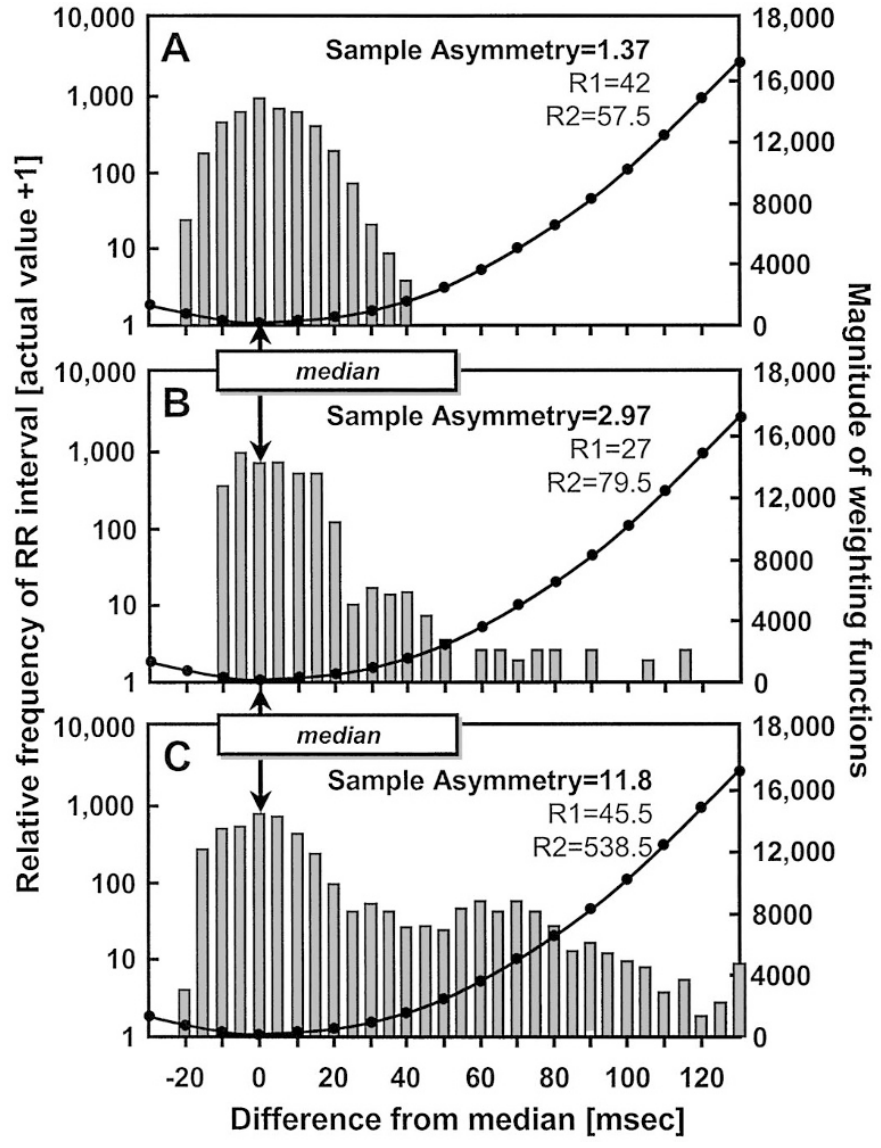

Figure 3. The frequency histograms of the time series shown in Figure 1 are displayed with a logarithmic ordinate (left axis) to aid the display; 1 has been added to all bins. The smooth lines represent the weighting functions used to calculate sample asymmetry (see Appendix). Sample asymmetry values as well as the values for the weighted deviations $R_{1}$ and $R_{2}$ are presented as well. Sample asymmetry rises dramatically before the catastrophic event.

Sample asymmetry analysis of simulated data. To confirm the ability of sample asymmetry to report in a graded fashion on HR time series with varying degrees of transient decelerations, we constructed simulations consisting of a baseline signal with the same frequency components as clinically observed neonatal HR data to which we added scaled versions of a clinically observed deceleration. In this way, we were able to simulate a wide range of abnormality of HRC. Figure $4 \mathrm{~A}$ shows an example of simulated data with baseline SD $7 \mathrm{~ms}$ and four decelerations of magnitude $100 \mathrm{~ms}$. This corresponds to moderately reduced HR variability with subclinical decelerations from $150 \mathrm{bpm}$ (RR interval $400 \mathrm{~ms}$ ) to $120 \mathrm{bpm}$ (RR interval $500 \mathrm{~ms}$ ). This is a realistic simulation-inspection of Figure $2 B$ suggests that a deceleration might easily be 20 -fold larger than the SD of a stable and low variability epoch of neonatal HR.

The SAA results are plotted in Figure $4 B$. Sample asymmetry is plotted as a function of the height of the deceleration measured in SD of the baseline signal. Figure 4 shows that more frequent and larger decelerations lead to increasing values of sample asymmetry, with values exceeding 10 in many conditions. For the example in Figure 4A, with four decelerations of height $14 \mathrm{SD}$, sample asymmetry is 5.7. 

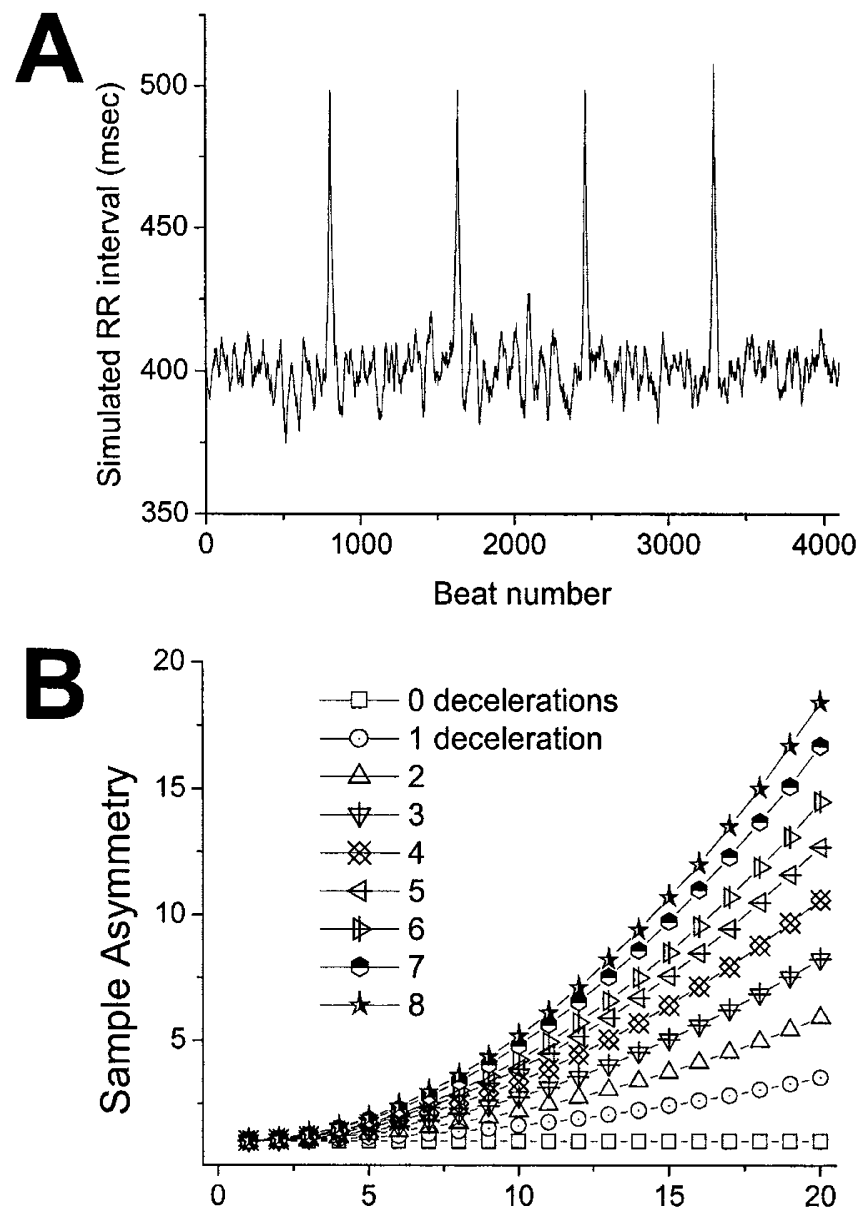

Height of deceleration (S.D. of baseline)

Figure 4. Decelerations increase sample asymmetry of simulated neonatal HR data. The simulated data were composed with a baseline process with the frequency content of normal neonatal HR records, and scaled versions of a clinically observed deceleration were added. (A) Simulated data series of length 4096 with baseline SD and decelerations chosen to mimic a moderately abnormal neonatal HR record. $(B)$ There is a monotonic increase in sample asymmetry with larger and more frequent decelerations. Each data point is the average of 100 realizations.
Population results. There were 38 positive blood cultures, growing coagulase-negative Staphylococcus $(n=12)$, Staphylococcus aureus $(n=7)$, Candida $(n=5)$, Klebsiella $(n=4)$, E. coli $(n=4)$, group B streptococcus $(n=2)$, Enterococcus $(n=2)$, Pseudomonas $(n=1)$, and Enterobacter $(n=1)$. Results from episodes with positive and negative blood cultures were pooled, as our prior analysis of HRC before the clinical diagnosis of sepsis and SIRS showed no difference between these groups (13).

Table $2 A$ shows the repeated measures ANOVA results for sample asymmetry values for experimental-group infants in 24-h blocks beginning $5 \mathrm{~d}$ before sepsis as well as for an average 24-h period post treatment. It is evident that sample asymmetry began increasing approximately $3-4 \mathrm{~d}$ before sepsis, with a steepest increase in the last $24 \mathrm{~h}$. The contrast between the baseline ( $5 \mathrm{~d}$ before sepsis) and the last $24 \mathrm{~h}$ was significant, $\mathrm{F}=5.5, p=0.02$. Within the experimental group, analysis of the contribution of accelerations (measured as the natural logarithm of $\mathrm{R}_{1}$ ) and of decelerations (natural logarithm of $\mathrm{R}_{2}$ ) led to the surprising finding that there was a significant fall in $\mathrm{R}_{1}(\mathrm{~F}=5.8, p=0.02)$ before sepsis, but no significant change in $\mathrm{R}_{2}$ (the last two columns in Table $2 A$ ). This is readily interpreted to mean that a decrease in the extent and duration of HR accelerations is more marked than increase in decelerations, which are more easily identified by eye.

As presented in Table $2 B$, post-treatment sample asymmetry returned to exactly presepsis levels. A contrast of the two periods of health, $5 \mathrm{~d}$ before sepsis versus post-treatment, showed no difference, $\mathrm{F}=0.01, p>0.99$. Similar return toward baseline was observed for both $\mathrm{R}_{1}$ (accelerations) and $\mathrm{R}_{2}$ (decelerations), although the second index, $\mathrm{R}_{2}$, remained somewhat elevated. As expected, a comparison of experimental-group infants' data in health to control-group infants revealed no significant difference in sample asymmetry $(\mathrm{F}=2.4$, $p=0.13)$ and no significance of the covariates BW and GA ( $p$ values of 0.2 and 0.7 , respectively). Similarly, no significant differences in $R_{1}$ and $R_{2}$ were observed between healthy and experimental infants in health (Table $2 B$ ).

Table 2. Sample asymmetry of heart rate, and asymmetries due to accelerations and decelerations before neonatal sepsis and SIRS, and in health

\begin{tabular}{|c|c|c|c|}
\hline & $\begin{array}{l}\text { Sample asymmetry } \\
\text { mean (SD) }\end{array}$ & $\ln \left(\mathrm{R}_{1}\right)$ (accelerations) & $\ln \left(\mathrm{R}_{2}\right)$ (decelerations) \\
\hline \multicolumn{4}{|l|}{ A: Changes before sepsis } \\
\hline $5 \mathrm{~d}$ before sepsis & $3.28(1.59)$ & $4.40(0.94)$ & $4.41(0.77)$ \\
\hline $3 \mathrm{~d}$ before sepsis & $3.50(1.94)$ & $4.27(0.92)$ & $4.39(0.80)$ \\
\hline $2 \mathrm{~d}$ before sepsis & $3.66(2.12)$ & $4.19(0.85)$ & $4.37(0.71)$ \\
\hline $1 \mathrm{~d}$ before sepsis & $4.20(2.32)$ & $4.10(0.87)$ & $4.28(0.65)$ \\
\hline Infants with sepsis posttreatment & $3.28(1.34)$ & $4.60(0.87)$ & $4.65(0.74)$ \\
\hline Contrast 2: $5 \mathrm{~d}$ before sepsis $v s$ post-treatment/recovery & $0.01, p>0.99(\mathrm{NS})$ & $2.1, p=0.2(\mathrm{NS})$ & $4.2, p=0.05$ \\
\hline Control infants & $2.90(0.93)$ & $4.75(0.67)$ & $4.67(0.62)$ \\
\hline Sepsis $v s$ control-ANOVA with covariates BW and GA* & $2.40, p=0.13(\mathrm{NS})$ & $0.35, p=0.6(\mathrm{NS})$ & $0.01, p>0.9(\mathrm{NS})$ \\
\hline
\end{tabular}

* Both covariates are not significant, $p$ values of 0.2 for BW and 0.7 for GA. 


\section{DISCUSSION}

A continuous monitoring procedure for detecting the early stages of neonatal sepsis and SIRS would be a useful adjunct to clinical care of this large and particularly vulnerable group of patients. In this regard, monitoring for abnormal HRC of reduced variability, specifically reduced accelerations, and transient decelerations observed in RR interval time series of infants destined for clinical illness may hold substantial promise.

Our original analytical approach was based on the finding that the characteristic abnormality of the RR interval time series led to asymmetric frequency histograms of the intervals, with a truncation of the left side, due to relative absence of accelerations, and enlargement of the right side, due to the frequency and abundance of decelerations (13). We here present the sample asymmetry, a new measure of the quantities of interest. sample asymmetry measures are not altered by missing points or by the ordering of the RR intervals. The method is based on weighting individual deviations of RR intervals from the median value and is conceptually similar to the low blood glucose index that we previously introduced and validated as a predictor of the risk for severe hypoglycemia in patients with type I diabetes $(15,16)$. For our analyses, we chose a quadratic weighting around the median, which is the most natural and theoretically warranted initial approach.

SAA is particularly well suited to our study as $R_{1}$ and $R_{2}$ can be directly interpreted as representing the frequency and extent of HR accelerations and decelerations, respectively. Thus, the numerical analysis can be immediately applied to an understanding of the pathophysiology of HR control. The finding in this study that the reduction of accelerations contributes more to the diagnostic abnormality of HRC than the more visually striking increase in decelerations is entirely novel. It points the way to more basic studies to test the hypothesis that the sympathetic nervous system activity is maximized before the clinical diagnosis of neonatal sepsis.

The mechanism of the abnormal HRC is not known. In addition to the current lack of a clear physiologic explanation, these abnormalities are not easily detected by the SD (13), which only detects low variability, or by frequency domain analysis (17), as the decelerations are intermittent but not periodic. Further limitations of frequency domain analysis include the unevenly sampled nature of the data, and the lack of assurance that every beat is captured. Thus, the quantification of the observed phenomena required development of novel quantitative methods, and empirical testing of their ability to detect neonatal sepsis and SIRS before clinical signs.

The clinical population reported here differs from our prior studies $(13,14,17,18)$. First, we prospectively collected clinical information, including duration of antibiotic therapy. Second, we noted specifically whether blood cultures were obtained for the clinical suspicion of sepsis and SIRS. These design changes allow more certain identification of episodes of clinical episodes of sepsis and SIRS.

Although SAA may prove to be useful tool in detecting early stages of neonatal sepsis and SIRS, it has limitations. For example, it will not detect epochs of reduced variability alone.
This limitation is important, as the classical abnormality of HRV in adults after acute myocardial infarction is simply reduced variability (19). In addition, it holds no information about the ordering of the intervals, and would not differentiate periodic from nonperiodic signals. In our group data, sample asymmetry was significantly elevated immediately before sepsis, but was also marked by a significant between-subject variability. Consequently, establishing a clear threshold value for onset of sepsis and SIRS that would be valid for all infants may not be feasible. Instead, we anticipate that potential clinical applications of sample asymmetry would be based on individual changes from baseline, or use other measures. Toward that end, we have recently found (18) that sample asymmetry adds independent information to other measures such as SD and sample entropy $(14,20)$ in identifying abnormal HRC with the all-important clinical goal of early detection of neonatal sepsis.

\section{REFERENCES}

1. Ventura SJ, Martin JA, Mathews TJ, Clarke SC 1996 Advance report of final natality statistics. Mon Vital Stat Rep 44:1-88

2. Stoll BJ, Gordon T, Korones SB, Shankaran S, Tyson JE, Bauer CR 1996 Late-onset sepsis in very low birth weight neonates: a report from the National Institute of Child Health and Human Development Neonatal Research Network. J Pediatr 129:63-71

3. Gray JE, Richardson DK, McCormick MC, Goldmann DA 1995 Coagulase-negative staphylococcal bacteremia among very low birth weight infants: relation to admission illness severity, resource use, and outcome. Pediatrics 95:225-230

4. Members of the ACCP/SCCM Consensus Conference Committee, American College of Chest Physicians/Society of Critical Care Medicine Consensus Conference 1992 Definitions for sepsis and organ failure and guidelines for the use of innovative therapies in sepsis. Crit Care Med 20:864-874

5. Bone RC, Grodzin CJ, Balk RA 1997 Sepsis: a new hypothesis for pathogenesis of the disease process. Chest 112:235-243

6. Gladstone IM, Ehrenkrantz RA, Edberg SC, Baltimore RS 1990 A ten-year review of neonatal sepsis and comparison with the previous fifty year experience. Pediatr Infect Dis J 9:819-825

7. Moro ML, DeToni A, Stolfi I, Carrieri MP, Braga M, Zunin C 1996 Risk factors for nosocomial sepsis in newborn infants and intermediate care units. Eur J Pediatr 155:315-322

8. Escobar GJ 1999 The neonatal "sepsis work-up": personal reflections on the development of an evidence-based approach toward newborn infections in a managed care organization. Pediatrics 103:360-373

9. Gerdes JS, Polin RA 1987 Sepsis screen in neonates with evaluation of plasma fibronectin. Pediatr Infect Dis J 6:443-446

10. Kaftan H, Kinney JS 1998 Early onset neonatal bacterial infections. Semin Perinatol 22:15-24

11. Aronson MD Bor DH 1987 Blood cultures. Ann Intern Med 106:246-253

12. Kellogg JA, Ferrentino FL, Goodstein MH, Liss J, Shapiro SL, Bankert DA 1997 Frequency of low level bacteremia in infants from birth to two months of age. Pediatr Infect Dis J 16:381-385

13. Griffin MP, Moorman JR 2001 Toward the early diagnosis of neonatal sepsis and sepsis-like illness using novel heart rate analysis. Pediatrics 107:97-104

14. Lake DE, Richman JS, Griffin MP, Moorman JR 2002 Sample entropy analysis of neonatal heart rate variability. Am J Physiol 283:R789-R797

15. Kovatchev BP, Cox DJ, Gonder-Frederick LA, Young-Hyman D, Schlundt D, Clarke WL 1998 Assessment of risk for severe hypoglycemia among adults with IDDM: validation of the low blood glucose index. Diabetes Care 21:1870-1875

16. Kovatchev BP, Straume M, Cox DJ, Farhy LS 2001 Risk analysis of blood glucose data: a quantitative approach to optimizing the control of insulin dependent diabetes. J Theor Med 3:1-10

17. Chang KL, Monahan KJ, Griffin MP, Lake DE, Moorman JR 2001 Evaluation of frequency domain methods for detection of sinus arrhythmia in neonatal heart rate time series. Ann Biomed Eng 29:764-774

18. Griffin MP, O'Shea TM, Bissonette EA, Harrell FE, Lake DE, Moorman JR Abnor$\mathrm{mal}$ heart rate characteristics preceding neonatal sepsis and sepsis-like illness. Pediatr Res 53:920-926

19. Kleiger RE, Miller JP, Bigger Jr JT, Moss AJ 1987 Decreased heart rate variability and its association with increased mortality after acute myocardial infarction. Am J Cardiol 59:256-262

20. Richman JS, Moorman JR 2000 Physiological time series analysis using approximate entropy and sample entropy. Am J Physiol 278:H2039-H2049 


\section{APPENDIX: SAMPLE ASYMMETRY OF A RANDOM VARIABLE}

To define sample asymmetry of a random variables we first introduce the following:

Weighting functions. Let $\xi$ be a random variable with values in its sampling space $X$ and unspecified distribution, and let $\mu$ $\epsilon X$ be a point within the sampling space $X$. For any $x \in X$ we define a weighting function $w(x ; \alpha)=(x-\mu) \alpha$, where $\alpha>$ 0 is a parameter describing the degree of weighting of deviations from the reference point $\mu$. For example, if $\alpha=1$, deviations from $\mu$ will receive linearly increasing weights, whereas if $\alpha=2$, deviations from $\mu$ will receive quadratically increasing weights. Note that the weighting parameter $\alpha$ could be selected in various applications to be any positive, including noninteger, number. A number smaller than 1 will result in slower than linear increase of weights, a number greater than 2 will result in a faster than quadratic increase of weights. Further, we define separate weighting for left and right deviations of \&xi; from its reference point $\mu$ as follows: 1) Leftweighting function: $w_{1}(x ; \alpha)=w(x ; \alpha)$ whenever $x<\mu$ and 0 otherwise, and 2) right-weighting function: $w_{2}(x ; \beta)=w(x ; \beta)$ whenever $x \geq \mu$ and 0 otherwise, where the parameter $\beta$, similarly to $\alpha$, describes the degree of weighting of deviation to the right of the reference point. To add flexibility to this model, we allow for different degree of weighting to the left $(\alpha)$ and to the right $(\beta)$ from the reference point $\mu$. In many applications, the left and right weightings could be equal (see Fig. 1).

Defining sample asymmetry of a random variable. Let $x_{1}$, $x_{2}, \ldots x_{\mathrm{n}}$ be a sample of $n$ observations on $\xi$. Given this sample, we define two quantities representing the sum of the weighted deviations to the left and to the right from the reference point $\mu$ as follows:

$$
\begin{aligned}
\mathrm{R}_{1}(\alpha)=\frac{1}{n} \sum_{i=1}^{n} w_{1}\left(x_{i} ; \alpha\right) \text { and } \\
\left.\mathrm{R}_{2}(\beta)=\frac{1}{n_{i=1}^{n}} \sum_{2} w_{2} ; \beta\right) \text {, respectively. }
\end{aligned}
$$

It is now clear that if $\alpha=\beta$, and the sample $x_{1}, x_{2}, \ldots x_{\mathrm{n}}$ is approximately symmetric with respect to the reference point, then $R_{1}$ will be approximately equal to $R_{2}$. If the sample is asymmetric with larger and/or more frequent deviations to the right from the reference point $\mu$, then $\mathrm{R}_{2}$ will be greater than $\mathrm{R}_{1}$. Inversely, if the sample is asymmetric with larger and/or more frequent deviations to the left from the reference point $\mu$, then $R_{1}$ will be greater than $R_{2}$.
Definition: The ratio

$$
\mathrm{R}(\alpha, \beta)=\frac{\mathrm{R}_{2}(\alpha)}{\mathrm{R}_{1}(\beta)}
$$

represents the sample asymmetry of the random variable .

The following properties are pertinent to the applications of sample asymmetry:

(a) If $\alpha=\beta$, when the sample $x_{1}, x_{2}, \ldots x_{\mathrm{n}}$ is approximately symmetric with respect to the reference point, then $\mathrm{R}(\alpha, \beta)$ will be approximately equal to 1 . Values greater than 1 will indicate larger and/or more frequent deviations to the right from the reference point $\mu$, whereas values less than 1 will indicate larger and/or more frequent deviations to the left.

(b) The sensitivity of the ratio $\mathrm{R}(\alpha, \beta)$ to left and right deviations from the reference point can be controlled through separate adjustment of the parameters $\alpha$ and $\beta$.

(c) $\mathrm{R}_{1}(\alpha)$ and $\mathrm{R}_{2}(\beta)$ can be used separately as estimates of the absolute weighted mass of the distribution of $\xi$; with respect to its reference point $\mu$.

(d) The reference point $\mu$ can be the empirical mean of the random variable $\xi$, e.g.

$$
\bar{x}=\frac{1}{n} \sum_{i=1}^{n} x_{i}
$$

the median of $\xi$, or any other theoretically or practically relevant number. The choice of a reference point could be critical for the subsequent data analyses. The median of the distribution is an obvious initial reference point because the median, as opposed to other statistics such as the mean, is not affected by outliers or large deviations in the data. This is particularly important in the analysis of RR intervals where the goal is to quantify spikes in the data.

(e) A quadratic weighting, that is, $\alpha=\beta=2$, has special properties that set it apart from other parameter configurations. First, the weighted deviations $\mathrm{R}_{1}(\alpha)$ and $\mathrm{R}_{2}(\beta)$ are exactly the squares of the classical Euclidean distances of the sample measured to the left and to the right from the reference point. Second, if the reference point $\mu$ is the mean (or the median) of the distribution, then, under the null hypothesis that the distribution is normal, the sample asymmetry $\mathrm{R}(2,2)$ will have an $\mathrm{F}$ distribution with $(n / 2-1 ; n / 2-1)$ degrees of freedom $(n / 2-1 / 2 ; n /$ $2-3 / 2$ if $n$ is an odd number). This property gives a straightforward statistical test for symmetry of a single data sample. Thus, we recommend the use of a quadratic weighting unless a particular data sample proves that another parameter combination is more appropriate. 